\title{
Correction to: Fabrication of high-strength polyoxymethylene fibers by gel spinning
}

Xudong Fang ${ }^{1,2,3,4}$ (D), Tom Wyatt ${ }^{2}$ (I), Jing Shi ${ }^{2}$ (D), and Donggang $\mathrm{Yao}^{2, *}$ (D)

${ }^{1}$ School of Mechanical Engineering, Xi'an Jiaotong University, 28 West Xianning Road, Xi'an 710049, Shaanxi, China

${ }^{2}$ School of Materials Science and Engineering, Georgia Institute of Technology, 801 Ferst Drive, Atlanta, GA 30332, USA

${ }^{3}$ State Key Laboratory for Manufacturing Systems Engineering, 99 Yanxiang Road, Xi'an 710054, Shaanxi, China

${ }^{4}$ State Key Laboratory of Robotics and System (HIT), 2 Yikuang Street, Harbin 150080, HeilongJiang, China

Published online:

13 June 2018

(C) Springer Science+Business

Media, LLC, part of Springer

Nature 2018

Correction to: J Mater Sci (2018) 53: 11901-11916

https://doi.org/10.1007/s10853-018-2410-5

In the original article, the name of author Donggang

Yao was misspelled. It is correct here.

The original article can be found online at https:/ / doi.org/10.1007/s10853-018-2410-5.

Address correspondence to E-mail: yao@gatech.edu 